Results Currently a work in progress and we are in the process of evaluating this work.

Conclusion A culture of engagement is more likely to predict performance than any other variables including competence (Alimo-Metcalfe \& Bradley, 2010). Through discussion and exploration we are able to expand upon learning and explore decision making. Disseminated learning allows for more openness and transparency amongst the team. There is a shift from the perception of a 'blame culture' to one of collaboration, inclusiveness and participation. Including staff who delivered the front-line care in these scenarios and discussions further enriches the process.

\section{P-160 THE BOTTOM LINE - NO PRESSURE!}

Anne Marie Jones. Garden House Hospice Care, Letchworth Garden City, UK

\subsection{6/bmispcare-2017-hospice.185}

Managing pressure injuries within palliative care patients has been shown to be challenging due to, decreased nutritional intake, reduced mobility and increasing frailty resulting from co-morbidities. Included in this would be patients taking steroids and those with complications from treatment which would compromise their skin integrity. Due to high numbers of patients being admitted to the inpatient unit with pressure injuries it was felt that reducing further deterioration of their pressure areas was paramount.

Aim The Tissue Viability Link nurse role is to initiate and incorporate creative ways to educate staff about the importance of preventing deterioration of pressure injuries in our patient group and keep staff up- to- date with the latest initiatives.

Method - A tissue viability focus group was developed to look at areas to improve the management of pressure injuries within the hospice

- A new policy has been devised incorporating the SSKIN algorithm and NICE guidelines

- Training sessions developed and delivered at nursing handovers and at away days

- A laminated pocket guide produced to enable staff to grade pressure injuries. An enlarged version of this was made for all the patient folders

- A patient and carer information leaflet produced about managing pressure injuries

- Heel mirrors purchased and informative sign produced so staff are reminded to use them

- Guidelines on type of wound dressings produced to streamline which dressings would be used

- 'The Bottom line' newsletter published bi-monthly covering relevant information for staff, interesting facts, 'Don't forget' section, 'News in Brief' section to keep staff updated

- SSKIN charts which are helping to prompt staff with repositioning our patients.

Results Mini audit and Hospice UK audits carried out have shown that the staff's knowledge and ability to recognise and put measures in place to minimise pressure injuries and complete necessary documentation has greatly improved.

Recommendations To continue to publish newsletter for staff and find innovative ways to raise staff awareness about ways to implement preventative measures. To continue to carry out Hospice UK audits within the allocated timeline.

\section{P-161 IMPROVING THE CONTENT AND TIMELINESS OF DISCHARGE LETTERS FROM A HOSPICE INPATIENT UNIT}

Joanne Rimmer, Aruna Hodgson. Wigan and Leigh Hospice, Greater Manchester, UK

\subsection{6/bmjspcare-2017-hospice. 186}

Background When a patient is discharged from an inpatient setting back into the community, essential information about their condition and its treatment must be communicated accurately and efficiently via a comprehensive discharge letter to the relevant health care professionals.

Aims To audit the content and timeliness of discharge letters from a hospice inpatient unit and rectify deficiencies identified by agreeing and implementing effective interventions.

To re-audit the impact of changes made, thereby completing the audit cycle.

Methods An initial audit identified that the standards for hospice discharge letters were not being met in several areas. Following this, four main strategies were implemented to achieve improvements:

1. Introduction of a discharge letter proforma

2. Enhanced communication systems between the medical team and clinical secretaries

3. Discharge letters faxed, rather than posted to ensure timeliness of delivery

4. Ongoing monitoring of when letters were being sent, with quarterly reports presented to the medical team.

One year following introduction of these changes, a retrospective re-audit was undertaken of twenty-five consecutive patients discharged from a hospice inpatient unit.

Results All areas of deficiencies previously identified were improved upon in the re-audit. The discharge letter proforma succeeded in standardising the format and content of letters, ensuring key positive and negative information was recorded. The systems put in place to ensure timeliness of letter-sending resulted in $96 \%$ being sent out within one working day, (versus $45 \%$ previously). These improvements have enhanced communication between the hospice, hospital and community.

Conclusions Further alterations to hospice discharge letters and ongoing monitoring are required to maintain and optimise standards. The audit work undertaken has demonstrated that effective clinical changes can be achieved through collaboration with colleagues to agree achievable action plans, and by ongoing good team working to attain high standards and additonal improvements in practice.

\section{P-162 ENHANCING DISCHARGE PROCESSES}

Tracy Parkinson, Janet McGeown. St Catherine's Hospice, Preston, UK

\subsection{6/bmjspcare-2017-hospice. 187}

Background Review of discharge processes was identified as a workstream within an overall in-patient unit project modernising ways of working to meet current service demands. Staff feedback showed increasing anxiety relating to completion of the discharge process and documentation, in particular relating to continuing health care funding. Anecdotally it was also felt that patient and family expectations of ongoing hospice care were a barrier to timely discharge planning.

Aims

1. Increase in number of patient discharges

2. Reduction in length of stay 
3. Increase staff understanding of discharge processes

4. Produce patient information leaflet.

Method A senior staff nurse led the work stream and undertook a retrospective base line audit of patient discharges and a staff survey to explore their experience of discharging patients from the in-patient unit (IPU). Using these results a patient leaflet has been produced, clearly outlining hospice expectations, discharge details and funding streams.A parttime discharge educator, with extensive experience of continuing health care, has been employed to review discharge processes and provide staff education. Education has been delivered one-to- one in relation to patient discharges in real time and within a formal setting.

Results Audit results have shown that the uncertainty of end of life care, family concerns and timely access to nursing home placements and care packages are significant factors when planning discharge from the IPU. It was also identified that clinical pressures affected timely completion of discharge documentation.

Achievements at six months:

- Increased patient discharges

- Reduction in average length of stay to 14 days

- All qualified staff have received training and awareness training for nursing assistants planned

- Patient leaflet completed.

Conclusion Patient flow and staff confidence have increased significantly following commencement of the project and the discharge educator post has been extended for an additional six months.

\section{P-163 USING ACUPUNCTURE TO TREAT HOT FLASHES AND NIGHT SWEATING FOR PATIENTS WITH BREAST CANCER}

Visweswaran Ramasamy. John Taylor Hospice, Birmingham, UK

\subsection{6/bmjspcare-2017-hospice. 188}

Background Hot flashes and night sweating are a common disruptive clinical problem that affects nearly two-thirds of all breast cancer survivors. Adjuvant hormonal therapies are an essential part of the treatment regimen for early breast cancer, and are used to prevent recurrence. However, hot flashes and night sweating are the most frequently occurring side effects of these interventions.

Aim To assess the effectiveness of acupuncture in breast cancer related hot flashes and sweating.

Methods A 44 year -old woman with a diagnosis of breast cancer had been suffering from intractable hot flashes and night sweating for the past two years. She had tried all other measures but these had very little or no effects on her symptoms. The subject received acupuncture treatment once a week for eight weeks, and the needles were stimulated manually every $10 \mathrm{~min}$ during each sessions. In all the treatment sessions, acupuncture points were stimulated manually and the needles were left in situ for 30 mins. Subjective measurement of vasomotor symptoms used Visual Analogue Scale (VAS).

Results A noticeable reduction in hot flashes and night sweating were observed after six sessions of treatment, which had a huge impact on her sleep pattern and psychological wellbeing.
By the end of eight weeks of treatment the subject reported a significant reduction in both her vasomotor and associated symptoms such as palpitations, anxiety, irritability and headaches.

Conclusion It would appear that eight sessions of acupuncture treatment had been shown to be effective in reducing vasomotor symptoms, especially for breast cancer patients who are seeking nonpharmacological therapies.

\section{P-164 THE ROLE OF KOREAN MEDICINE IN PALLIATIVE MEDICINE FOR PATIENTS WITH TERMINAL CANCER}

Hae-chang Yoon, Jung-hyo Cho. Dae-Jeon University, Daejeon, Republic of Korea

10.1136/bmjspcare-2017-hospice. 189

Background Modern palliative care began in the hospice movement led by Dr. Cicely Saunders. In Korea, it was started earlier but built the foundation recently regarding Korean medicine (KM). In 2015, the strategy for development of KM was established. It included the guidelines of cancer-related fatigue and anorexia.

Objectives The aim was to figure out the current state of hospice-palliative care (HPC) and evaluate the effectiveness of $\mathrm{KM}$ in terms of HPC.

Methods Systemic reviews published until Feb 2017. Literatures in international journals were searched on PubMed, MEDLINE, ScienceDirect and CINAHL; and articles in Korean journal on OASIS operated by the Korean Institute of Oriental Medicine. Keywords were palliative care with acupuncture, moxibustion and herb medicine in English and Korean

Results The results in acupuncture, moxibustion and herb medicine were 19,3 and 15 respectively. First, acupuncture was effective to relieve nausea and vomiting, anxiety, cancerrelated fatigue and leukopenia. Second, moxibustion was helpful for treating nausea and vomiting, cancer-related fatigue and leukopenia. Third, herb medicine was beneficial to improve quality of life and make the scales of immune system better. But there were some conflictive outcomes about other symptoms. Nevertheless, our view was there was no or slight adverse effect and most patients treated with KM were satisfied. Recently the number of hospitals and medical staff for HPC has been increasing but not enough compared with the number of patients who need HPC. Additionally the number of patients using $\mathrm{KM}$ has grown and the classification of them varied but the National Health Insurance didn't cover it sufficiently.

Conclusion The result of this review suggested that $\mathrm{KM}$ was effective for some symptoms related to terminal cancer patients. Therefore it may be the appropriate treatment for palliative care. This is the reason why we should make effort for $\mathrm{KM}$ to be more developed for end-of-life patients.

\section{P-165 SEPSIS CARE BUNDLE FOR A HOSPICE IN-PATIENT UNIT}

Paula Powell, Daniel Monnery, Susan Schofield. Willowbrook Hospice, Merseyside UK

10.1136/bmispcare-2017-hospice. 190 